\title{
Algorithms for Opportunistic Load Balancing Cognitive Engine
}

\author{
Marko Mäkeläinen, Zaheer Khan, Tuomo Hänninen, and Harri Saarnisaari \\ Centre for Wireless Communications \\ Department of Communication Engineering \\ University of Oulu \\ P.O.Box 4500, 90014-Oulu Finland \\ Email: \{marko.makelainen, zaheer.khan, tuomo.hanninen, harri.saarnisaari\}@ee.oulu.fi
}

\begin{abstract}
This paper presents a cognitive engine (CE) that intelligently and dynamically allocates spectrum resources to users in the following two cases: 1) a scenario where a network has an exclusive access to a spectrum band; and 2) a spectrum sharing scenario where along with the exclusive utilization to its own spectrum band a network also can opportunistically utilize shared spectrum band as a second user. Moreover, the implemented CE performs two main tasks: 1) Accepts or rejects arrival user requests based on a priority based algorithm; and 2) It intelligently load balances the user traffic between the two available network resources, while taking into account the primary user activity in the shared spectrum band. We evaluate performance of the proposed algorithms under different primary and secondary user traffic scenarios. We show that the proposed load balancing algorithm increases average throughput of the network and it also reduces the average number of users rejected by the network.
\end{abstract}

\section{INTRODUCTION}

The overall mobile data traffic per month will increase by multiple times by 2017 [1, Fig. 1]. The growth in mobile data traffic can increase the network congestion which may cause a risk of the network resource being overloaded under heavy utilization. As a consequence, the Quality-of-Service (QoS) for the network services may be difficult to maintain resulting in decrease of the end-user service quality.

In the case of cellular networks, possible solutions for alleviating network congestion are studied and experimented in recent past with varying success [2] [3]. Traditionally, the problem is solved by adding more cells in the congested areas. However, the radius of the cells in urban areas will eventually become so small that it may become impractical in the near future to add more macro cells to increase capacity [4]. One more proposed solution to the problem of the network congestion is the femtocell technology. Although femtocells are shown to improve cellular coverage, interference mitigation issues between macro and femtocells remain to pose serious challenges to their practical deployment [5]. Moreover, management complexity for the operator, QoS issues due to internet protocol (IP) backhauling, and unwillingness of users to pay additional data charges for internet back-end connectivity, add to the problem. In this way, the increase of capacity through load balancing may be invaluable. The aim of the load balancing is to share radio resources efficiently among the users. Through efficient capacity sharing the total capacity for serving user should increase.

Load balancing cellular traffic to existing infrastructures that provide wireless access has also been proposed to solve the congestion problem in the cellular networks [6] [7] [8]. One such example is load balancing of cellular traffic between cellular and Wi-Fi networks. In addition to the extensive coverage of existing Wi-Fi structures, the deployment of new $\mathrm{Wi}-\mathrm{Fi}$ access points to increase capacity and coverage is simple. However, Wi-Fi networks are operating on a best effort basis and they do not provide QoS guarantees, new techniques for reliable utilization of $\mathrm{Wi}-\mathrm{Fi}$ for offloading scenarios are required.

To help reduce the network congestion due to rising demand for high-bandwidth wireless data services, recently, both the Federal Communications Commission (FCC) and the European Union (EU) have recently recommended the adoption of spectrum sharing technologies [9] [10]. Under the spectrum sharing paradigm, the opportunistic load balancing of the cellular traffic to other underutilized networks provides an efficient solution for intelligent resource usage. One way to achieve efficient load balancing is to incorporate cognitive decision-making for a part of the current offloading system. Instead of balancing the traffic in an arbitrary way, the network system with cognitive decision-making is able to share the traffic load intelligently within and between networks to maximize the benefits of the multiple networks.

In this paper, we provide a novel solution for efficient load balancing among multiple network resources. The growth of the existing network infrastructure introduces a favorable environment to utilize the proposed load balancing scheme. In addition, the proposed solution is able to take advantage of new technologies and existing networks, such as pico cell (femtocell) technology, since user terminals are developing at a good pace. The rest of the paper is organized as follows. Section II describes the system model. In Section III represents our scheme as a solution for load balancing paradigm. In Section IV, the simulation parameters and the simulation results are represented. In Chapter 5, the conclusions are discussed.

\section{SYSTEM MODEL}

We consider a cognitive radio network (CRN) consisting of $\mathrm{R}$ network base stations and $\mathrm{N}$ number of network users. A user attempts to connect to the base station with a traffic

This research was done in the CORE+ project which was supported by VTT Technical Research Centre of Finland, University of Oulu, Centria University of Applied Sciences, Nokia Solutions and Networks, PehuTec, EXFO, Elektrobit, Anite, Rugged Tooling, Finnish Defence Forces, Finnish Communications Regulatory Authority, and Tekes the Finnish Funding Agency for Innovation. 
data rate demand $D$ defined as $\mathrm{kbit} / \mathrm{s}$. The user will leave and release the allocated resource when its holding time $T$ expires given that the base station accepted the connection request of that user. The potentially available accessible resources for a user are radio-frequency bands each of which are divided into $L$ number of resource slots providing the total bandwidth $B$ expressed as symbols/s in a radio channel as quite usual when data rates are discussed. As an example, the slots may be time division multiple-access (TDMA) slots. There are two kind of resources: 1) A dedicated spectrum band where resource slots are exclusively allocated to a particular system; and 2) A shared spectrum band where potentially available resource slots are opportunistically allocated without disturbing the primary user (PU). In the shared spectrum band, the occupancy status of a PU in each resource slot is obtained from a database. In different slots the occupancy of a PU may be different; in a given resource slot, the probability of the PU being present is $\theta_{i} \in[0,1)$. Moreover, the PU activity in a resource slot is independent of the PU activity in other resource slots. The PU occurrence probabilities are also contributed, such as the minimum probability is 0.1 at the beginning and the maximum is 0.5 at the end of the resource time frame. For example, if the total amount of time slots in one TDMA frame is 20, then the PU occurrence probability in slots 1 to 4 is 0.1 , slots 5 to 8 is 0.2 . Finally, from 17 th slot to 20 th slot the probability is 0.5 . The primary user occurrence probabilities are scaled in proportion to the number of time slots in a TDMA frame.

In the shared resource, we study the proposed load balancing algorithms under two different PU activity models: 1) the PU occupancy remain stationary; or 2) the PU occupancy may change after $t$ intervals. For evaluating the impact of PUs on the user allocation, we compare the performance of the system when PUs may be present to the scenario when there are no PUs in the system. In the variable PU occupancy model, a new PU arrangement is generated every tenth allocation.

As in the contemporary cellular systems, the user base of the networks is heterogeneous. Therefore, the diversity has to be taken into account in the simulator. There are five different user types in the simulator: an emergency call, a voice call, a short message service (SMS), a data transfer, a realtime video. We use different parameters to classify users: 1) Occurrence probability; 2) priority value; 3 ) data rate demand; 4) available modulation schemes, such as binary phase-shift keying (BPSK), quadrature phase-shift keying (QPSK), 16quadrature amplitude modulation (16QAM) and 64-quadrature amplitude modulation (64QAM); and 5) holding time which describes how long a user will need a resource until it is fully served or data to transmit value which describes how much data is left to transmit until the user is fully served. The load balancing cognitive engine (CE) utilizes the modulation scheme with the required data rate to calculate the number of resource slots to be assigned for a user.

When a user wants to transmit data, it requests resource slots from the network. In our model, we have $X$ different kinds of user traffic; some are delay tolerant, such as data users, and some don't tolerate any delay, such as voice call users. The holding time and the required data rate may vary among and between user types. In addition, some user types may have higher priority compared to other user types. For example, an emergency call user may have the highest priority value. The priority values and user demands are utilized to sort the users for the allocation. After sorting, if there are available resource

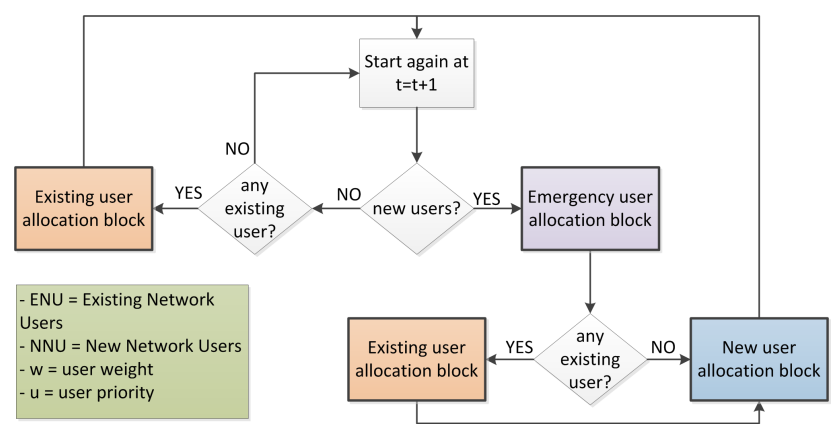

Fig. 1. Greedy algorithm flow chart for access to exclusive licensed band.

slots, the user will be allocated to the first available slots of the resource. Otherwise, the user will be dropped or placed in a queue for waiting the release of resources. The amount of resource slots by a user depends on the required data rate of the transmission; the provided transmission capacity of a single resource slot is $B / L$. For the scenarios where the network cannot fulfill the user request, we analyze the performance of our algorithms under two different models: 1) A rejected user is dropped by the network; and 2) A rejected user is only dropped if it's a voice user, otherwise, it is placed in the queue by the network. If a PU appears in a resource slot during the allocation process, the user that is allocated to that resource slot needs to be moved to some other slot by the network, if any available, otherwise the user is dropped by the network or placed in the queue.

\section{PROPOSED SOLUTIONS}

\section{A. A greedy algorithm for sorting users}

Since our system has different user traffic types, the algorithm for selecting a user to be allocated to the resource is needed. In order to solve the resource allocation problem faster and more efficiently, a greedy algorithm is used for sorting [11] [12].

The greedy algorithm utilized for sorting of the users for the considered allocation problem is explained as follows. The priority value $u_{n}$ represents user's importance. The greater user's priority value is the greater importance user has. The highest priority values could be assigned to the emergency call or to the possible control signals of the network users. Within the weight and the priority value the greedy ratio (GR) is formulated. The greedy ratio $G R$ is defined as

$$
G R_{n}=\frac{u_{n}}{w_{n}},
$$

where $u_{n}$ is the importance of the user $n$, the $w_{n}$ is the weight of the user (number of resource slots demand). The users currently sending data are pooled together into the load balancing process in order to be allocated by the greedy algorithm. In the algorithm, the gathered users are sorted in the following order by the greedy ratio value:

$$
\frac{u_{1}}{w_{1}} \geq \frac{u_{2}}{w_{2}} \geq \ldots \geq \frac{u_{n}}{w_{n}} .
$$

\section{B. Dynamic resource allocation for exclusive licensed band}

Decision flow diagram of resource allocation algorithm for the scenario where the network can utilize its own exclusive resource only is illustrated in Figure 1. In the flow chart, $u$ stands for the priority of the user, and $w$ is the weight of the 


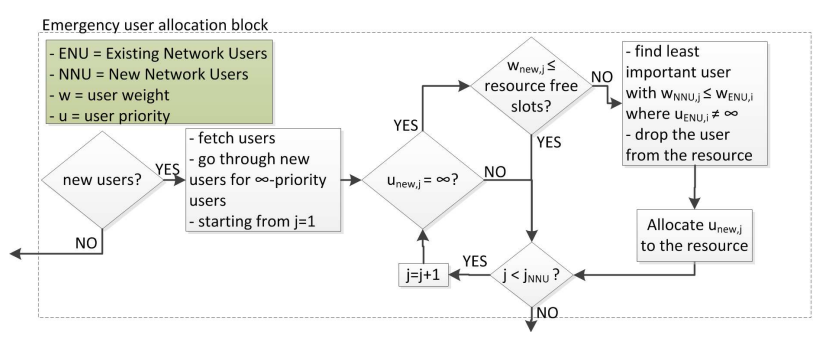

Fig. 2. Emergency user procedure flow chart for access to exclusive licensed band.

user. During the allocation process, the users of the network are classified as new network users (NNU) and existing network users (ENU). The algorithm has two separate processes: Continue serving existing users present in the network, and allocating new arriving users. To incorporate changes in the user demands in the network, the dynamic resource allocation process is iterated every $t$ second. In each simulation, the interval $t$ is 1 second.

1) User arrival and departure: The arrival of users in every iteration follows a random process; that is a given number of users will appear in a given time period. The simulation parameter tables, described in simulation result section, will describe the used arrival parameters of each simulation. In a given iteration, the arrival of users will a initiate emergency user allocation procedure, if any, otherwise, it initiates nonemergency user allocation process. These procedures are explained next.

2) Emergency user allocation: The process begins by examining priority levels of new users. If an emergency user arrives, the algorithm checks whether there are any available free resource slots in the resource. If the number of free slots in the resource is greater than or equal to the slot demand of the user, no existing user dropping decisions are needed because the user will have the required slots. Otherwise, the least important user with greater or equal slot demand will be dropped from the resource and the emergency user will be allocated into the released resource slots. After all new users are examined, the algorithm will move to the existing user allocation procedure if there are existing users in the system. Figure 2 represents the emergency user allocation procedure.

3) Existing user allocation update: After the actions for new emergency users are completed, the algorithm checks whether there are any existing users in the resource. The algorithm will go through all allocated users and undertakes necessary routines. The first task is to check whether the user is fully served. The timeout of holding time indicates that the user has completed all of its transmissions and it won't need the resource anymore. Therefore, the user will be deactivated and removed from the resource. Moreover, the capacity of the current resource will be increased by the weight $w_{i}$ of the user $i$. If the holding time of the user has not expired, the following procedure will be performed: 1) The holding time will be decreased by the elapsed time since the last routine; and 2) the capacity of the allocated resource will be kept unchanged. Correspondingly, the holding time of the data user will be reduced by the data sent during the time interval. This procedure will be held for all allocated users. Figure 3 represents the existing user allocation procedure.

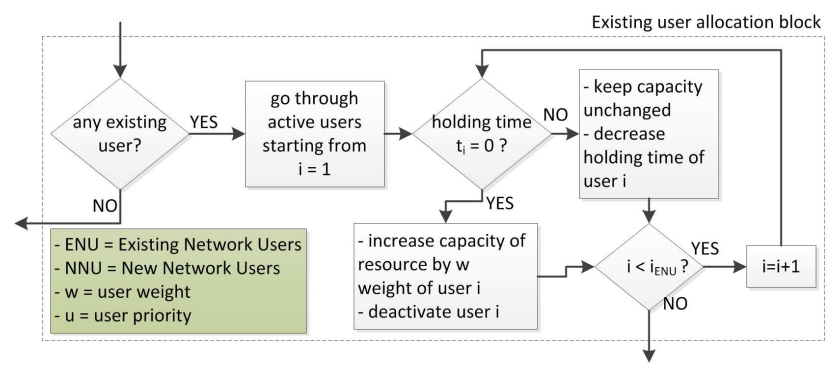

Fig. 3. Existing user procedure flow chart for access to exclusive licensed band.

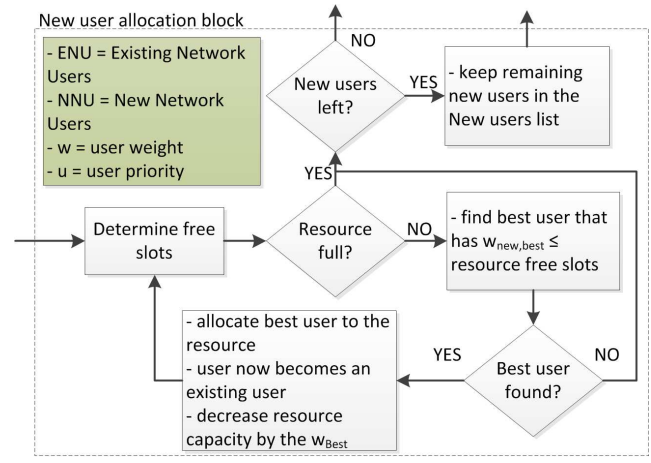

Fig. 4. Non-emergency user procedure flow chart for access to exclusive licensed band.

4) Non-emergency user allocation: At the beginning, the algorithm calculates the largest number of consecutive free time slots and filters users that requires less or equal amount of time slots, from which the most valuable user is selected by the greedy algorithm described earlier. This process will be repeated as long as all new users are not allocated, given that there are available free time slots for the allocation. Users who were left unallocated will be queued for the next allocation round. However, if the user cannot tolerate queuing (such as a voice call user), it will be dropped instead. Figure 4 represents the non-emergency user allocation procedure.

\section{Dynamic resource allocation for opportunistic spectrum sharing}

While operating with a shared resource along with the exclusive licensed band, the described algorithm needs to be modified to comply with the principles of opportunistic sharing. The basis of the opportunistic algorithm is similar to the exclusive licensed band case with the following functionalities.

At the beginning of the iteration, the algorithm checks whether the primary user information has changed. Moreover, changes in the PU occupancy on opportunistic resources initiate a primary user routine. The primary user routine removes all allocated users from the opportunistic shared resource and adds them to a moved user list to wait for re-allocation. The reallocation of the users occurs in the allocation procedure of the non-emergency users. The voice call users are also placed temporally in the moved user queue for reallocation. However, they will be dropped unless they managed to access the resources immediately in the next allocation. In Fig. 5 the effect of the primary user occupancy behavior is illustrated.

After the primary user process, the algorithm checks whether there are any new or moved users. If a new user 


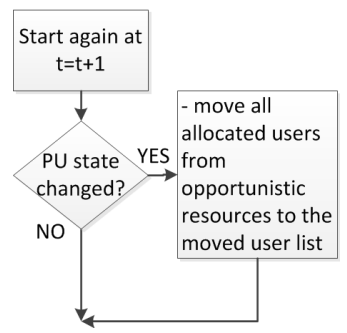

Fig. 5. The routine for new primary user appearance as a flow chart.

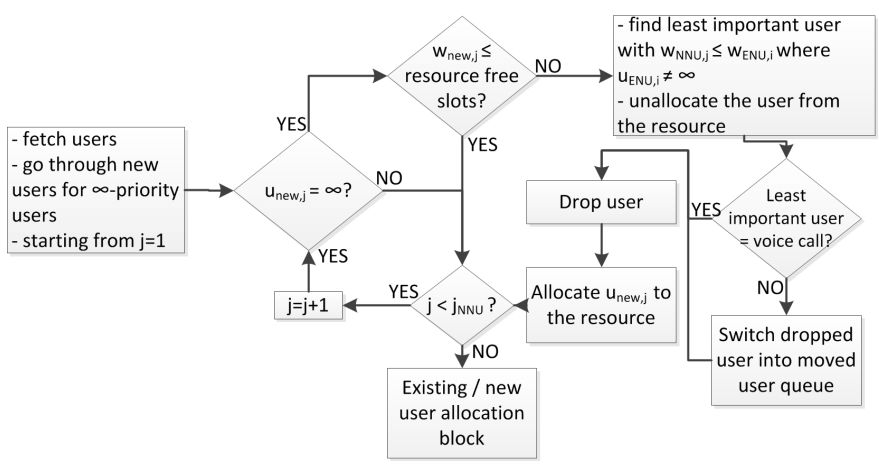

Fig. 6. The flow chart of the priority check procedure for the exclusive licensed band plus a shared band.

is found, the algorithm executes the priority search for each new user. If any emergency user is found, the algorithm checks whether there are free slots in the resource. In case where an emergency user is found and the main resource is full, the algorithm has to deallocate the least important user from the main resource and allocate the emergency user to the resource. The deallocation operates as in the exclusive resource algorithm, since the algorithm is able to queue the least important user in moved user list instead of dropping it. The priority check routine is illustrated in Fig. 6 .

The existence of moved or new users will start the nonemergency user allocation. First, the allocation in the exclusive licensed band is executed. The allocation process is the same than in the exclusive licensed band algorithm. After it is fully occupied, the algorithm begins to allocate the users in the shared band, if any requesting users are remaining and if any free resource slots are available in the opportunistic resource. Therefore, the first resource to be utilized is the own exclusive licensed band $(\mathrm{r}=1)$. The reallocation of moved users follows the same principles as the new user allocation process. After the moved users have been examined, the algorithm goes through the new users as described before. The allocation procedure for the exclusive licensed band plus a shared band is presented in Figure 7.

\section{Simulation Results}

A. Cognitive engine based load balancing: Benefit analysis

Benefit analysis of the proposed opportunistic CE based load balancing is presented in this section. A network is first simulated independently, i.e., without opportunistic utilization of the other resource, and then in a load balancing scenario, where the network can load balance between its own resource and the opportunistic resource. We use the following two concepts to evaluate the performance of the proposed solution.

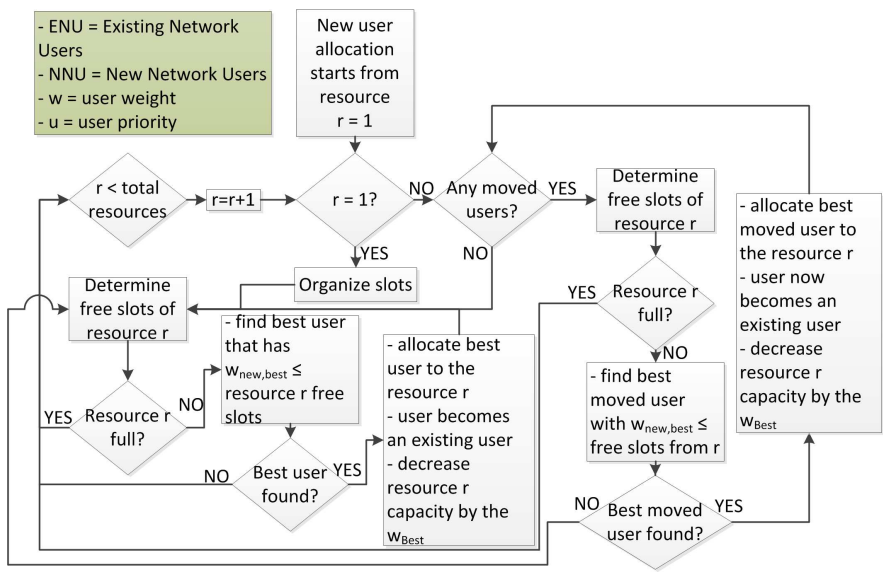

Fig. 7. Allocation procedure for the exclusive licensed band plus a shared band.

TABLE I. THE SIMULATION PARAMETERS.

\begin{tabular}{|l|c|}
\hline Parameter & value(s) \\
\hline Number of resources & 2 \\
Resource capacity in BPSK & $1000 \mathrm{kbps}$ \\
Slots / resource & 20 \\
Simulated users & $40,60,80,100$ \\
User arrivals & $0-3$ per second \\
Simulation time & 300 seconds \\
Simulation count & 10000 times in each settings \\
Queueing scenario & queueing \& non-queueing \\
\hline Simulated PU scenarios & single resource \\
& two resources - no PU \\
& two resources - variable PU \\
& two resources - stationary PU \\
\hline
\end{tabular}

1) Load balancing throughput gain: The load balancing throughput gain is defined as follows:

$$
G_{T}=\frac{\sum T_{1 \& 2(O P)}-\sum T_{1(I N D)}}{\sum T_{1(I N D)}},
$$

where $T_{1(I N D)}$ is throughput of the network without load balancing and $T_{1 \& 2(O P)}$ is the throughput with opportunistic load balancing.

2) Reduction: Reduction is blocking due to load balancing as compared to when an exclusive licensed band is utilized. Reduction is given as

$$
B R_{T}=\frac{B R_{1(I N D)}}{B R_{1 \& 2(O P)}},
$$

where $B R_{1(I N D)}$ is the number of rejections when an exclusive licensed band is utilized and $B R_{1 \& 2(O P)}$ represents the number of rejections when a main resource plus an opportunistic resource is utilized.

\section{B. Impact of the increased number of users on the system}

In this simulation, we generate different amount of users: $40,60,80$, and 100 users. The simulation process is performed separately for each number of users in order to achieve comparable results. The purpose of the chosen user amounts for the simulations are to test the network system on different traffic loads. The simulation parameters are represented in Table I and Table II.

Figure 8 illustrates how throughput gain behaves as a function of the number of users in the simulation scenarios. The achieved gain of utilizing the opportunistic resource depends 

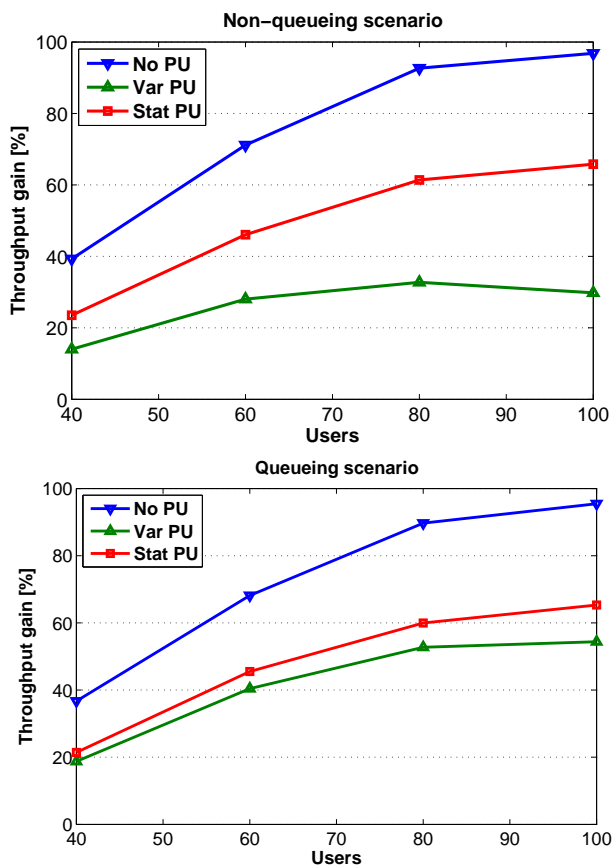

Fig. 8. Throughput gain of using the opportunistic resource under different PU activity schemes as a function of users.

TABLE II. USER PARAMETERS.

\begin{tabular}{|l|c|c|c|c|c|}
\hline User Type (t) & Emergency call & Voice call & SMS & Data & Real-time video \\
\hline Probability & 0.001 & 0.2245 & 0.2245 & 0.45 & 0.10 \\
\hline Priority & $\infty$ & 3 & 3 & 1 & 1 \\
\hline Traffic load & light & light & light & medium & heavy \\
\hline Data rate (kbps) & 64 & 13 & - & - & $100-600$ \\
\hline Modulation & BPSK, QPSK & QPSK & all & all & all \\
\hline Duration or & $50-110$ & $90-360$ & $1 \mathrm{kbit}$ & $\begin{array}{c}100-1000 \\
\text { kbit }\end{array}$ & $\begin{array}{c}150-350 \\
\text { seconds }\end{array}$ \\
data to transmit & seconds & seconds & & &
\end{tabular}

heavily on the behavior of the primary users. Even if the amount of available free opportunistic slots in the stationary case and the variable case are equal in average, the gain is more than twice when the PUs are stationary. This is due to the allocation of a heavy user to the opportunistic resource. A heavy user, herein a video or a data user, may require multiple slots for transmission. Sometimes the situation may appear promising to allocate a heavy user in the opportunistic resource, but in the very next moment the allocation could be impossible to maintain due to the increased PU amount. The absence of predictability of variable PU scenario might cause difficulties for the algorithm allocate users for long periods of time.

Figure 9 represents the blocking reduction as a function of the number of users. Only the non-queueing scenario plot is represented because the queueing scenario plot is almost equal. It can be seen that the reduction is strongest with smaller number of user when there are no primary users utilizing the resource. Therefore, there are more free resources available for the proposed opportunistic utilization. The differences between PU activity scenarios decreases as the amount of users increases in the system. Moreover, the reduction of the variable primary user scenarios is becoming slightly better than in the other PU scenarios while the congestion increases in the system.



Fig. 9. Blocking reduction of using the opportunistic resource under different PU activity schemes as a function of users.

TABLE III. THE SIMULATION PARAMETERS.

\begin{tabular}{|l|c|}
\hline Parameter & value(s) \\
\hline Number of resources & 2 \\
Resource capacity in BPSK & $1000 \mathrm{kbps}$ \\
Slots / resource & 20 \\
Simulated users & 240 \\
User arrivals & 4 per minute \\
Simulation time & 3600 seconds \\
Simulation count & 10000 times in each settings \\
Queueing scenario & queueing \& non-queueing \\
\hline Simulated PU scenarios & single resource \\
& two resources - no PU \\
& two resources - variable PU \\
& two resources - stationary PU \\
\hline
\end{tabular}

\section{Emergency call impacts on system performance}

In this simulation we study the network behavior in the state of emergency by increased amount of emergency calls. The simulation parameters are represented in Table III. The emergency users will always be allocated to resources unless the resource is already fully occupied by other emergency users. Since emergency users are in a privileged position compared to the other users, this may have impact on the system performance.

We evaluate the system performance in five different user type occurrence probabilities. We increase the probability of emergency call users and compensate this by decreasing either the probability of data and video users. The user type occurrence probabilities utilized in the simulations are represented in Table IV. In this simulations, the holding time of a user takes an integer value and it is uniformly distributed between 1 and 5 minutes. The user parameters are represented in Table V.

In the simulations, the throughput gain decreases as the occurrence probability of a emergency call increases. The major reason for this phenomenon is the decrease of average

TABLE IV. THE USER TYPE OCCURRENCE PROBABILITIES.

\begin{tabular}{|c|c|c|c|}
\hline Case & Emergency call & Voice call & Data \& video \\
\hline 1 & 0,01 & 0,60 & 0,39 \\
2 & 0,05 & 0,60 & 0,35 \\
3 & 0,10 & 0,60 & 0,30 \\
4 & 0,15 & 0,60 & 0,25 \\
5 & 0,20 & 0,60 & 0,20 \\
\hline
\end{tabular}

TABLE V. USER PARAMETERS.

\begin{tabular}{|l|c|c|c|}
\hline User Type (t) & Emergency call & Voice call & Data \& video \\
\hline Probability & \multicolumn{4}{|c|}{ see Table IV } \\
\hline Priority & $\infty$ & 3 & 1 (data), 2 (video) \\
\hline Data rate (kbps) & 64 & 13 & $100,200,300,400,500$ or 600 \\
\hline Modulation & BPSK, QPSK & QPSK & all \\
\hline Holding time & \multicolumn{4}{|c|}{$1-5$ minutes } \\
\hline
\end{tabular}



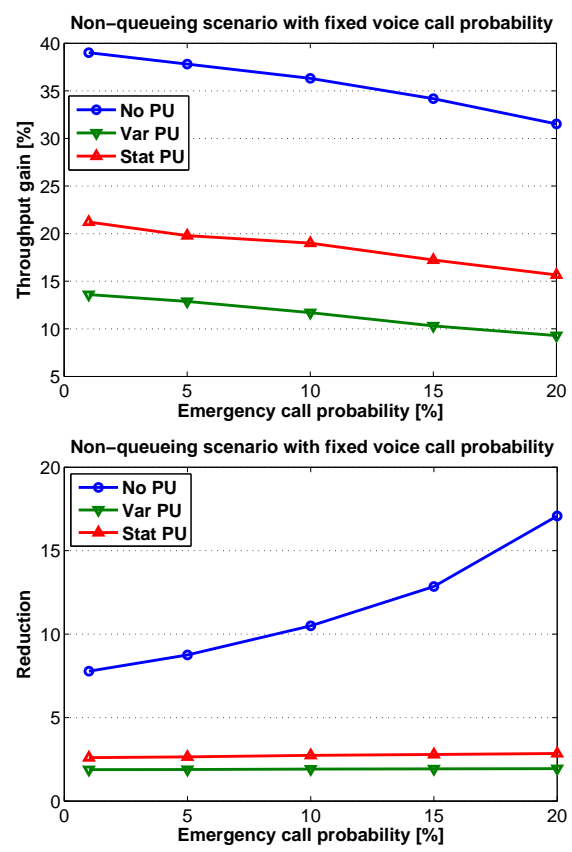

Fig. 10. Throughput gain and blocking reduction as a function of emergency call probability.

requests data rate per a user. Moreover, the average request data rate decreases due to the decrease of higher data rate requesting data and video users. Fig. 10 illustrates the throughput gain results.

As seen in this simulation, the reduction of blocking rate grows significantly in the no PU activity scheme. The remarkable growth of the reduction is due to the increased amount of emergency calls which are displacing the least important users from the resources. Moreover, the major reason is that the emergency call users require less capacity than the data and video users. In the others PU activity schemes, the increased congestion of the system due to the lack of free resources prevented the increase of the blocking reduction. Although the throughput decreases in the system as the occurrence probability of an emergency call increases, the value and utility of the network system remain precious in the state of emergency. The blocking reduction results are represented in Figure 10.

\section{Conclusions}

In this work, we proposed resource allocation schemes for dynamic and opportunistic wireless networks. We considered two different resource allocation scenarios 1) when only one exclusively owned spectrum band is available; and 2) when along with the exclusively owned band there is also a spectrum band that can be utilized opportunistically. We also considered three PU activity schemes for the opportunistic spectrum access; 1) no PU activity; 2) stationary PU activity; and 3) varying PU activity.

We have presented throughput gain and blocking reduction of the user to evaluate the performance of the proposed scheme under two simulation schemes: 1) impact of increased user amount; and 2) emergency call impacts on the system performance. We found that using our scheme in spectrum sharing can result in throughput gain of up to 30 percent when the occupancy behavior of the PU is varying during the running of the algorithm. Moreover, the gain of up to 65 percent when the primary user remain stationary. Our results also show that when the users are queued the the throughput gain is significantly increased for the case of varying primary users. We also show that our scheme reduces the blocking of the users up to four times.

\section{REFERENCES}

[1] "Cisco visual networking index: Global mobile data traffic forecast update, 2012-2017 (accessed 21.12.2013)," uRL: http://www.cisco.com/en/US/solutions/collateral/ns341/ns525/ns537/ ns705/ns827/white_paper_c11-520862.pdf.

[2] T.-A. Ngo, S. Perreau, and A. Dadej, "Releasing congestion in next generation cellular networks by using static channel relaying strategy analytical approach," in Networks, 2005. Jointly held with the 2005 IEEE 7th Malaysia International Conference on Communication., 2005 13th IEEE International Conference on, vol. 1, 0-0 2005, p. 6 pp.

[3] N. Marathe, G. Biradar, U. Desai, and S. Merchant, "A comprehensive call management strategy for congestion control in cellular networks," in Communication Systems Software and Middleware, 2007. COMSWARE 2007. 2nd International Conference on, jan. 2007, pp. $1-7$.

[4] "Deployment strategies for heterogeneous networks, white paper (accessed 13.01.2013)," uRL: http://www.nokiasiemensnetworks. com/sites/default/files/document/20120531_nokia_siemens_networks_ deployment_strategies_for_heterogeneous_networks_final.pdf.

[5] G. Gü andr, S. Bayhan, and F. Alagö andz, "Cognitive femtocell networks: an overlay architecture for localized dynamic spectrum access [dynamic spectrum management]," Wireless Communications, IEEE, vol. 17, no. 4, pp. $62-70$, august 2010.

[6] E. Yanmaz and O. Tonguz, "Dynamic load balancing and sharing performance of integrated wireless networks," Selected Areas in Communications, IEEE Journal on, vol. 22, no. 5, pp. 862 - 872, june 2004.

[7] S. Dimatteo, P. Hui, B. Han, and V. Li, "Cellular traffic offloading through wifi networks," in Mobile Adhoc and Sensor Systems (MASS), 2011 IEEE 8th International Conference on, oct. 2011, pp. $192-201$.

[8] B. Han, P. Hui, V. A. Kumar, M. V. Marathe, G. Pei, and A. Srinivasan, "Cellular traffic offloading through opportunistic communications: a case study," in Proceedings of the 5th ACM workshop on Challenged networks, ser. CHANTS '10. New York, NY, USA: ACM, 2010, pp. 31-38. [Online]. Available: http://doi.acm.org/10.1145/1859934.1859943

[9] Office of Engineering and Technology Wireless Telecommunications Bureau, "White spaces and spectrum sharing for wireless broadband," FCC Open Agenda Meeting: Progress Report, July 2012. [Online]. Available: http://transition.fcc.gov/files/presentations/ 2012-06-19_White_Space_Progress_Report.pdf

[10] EUROPEAN COMMISSION, "Communication from the commission to the european parliament, the council, the european economic and social committee and the committee of the regions promoting the shared use of radio spectrum resources in the internal market," September 2012, cOM(2012) 478 final. [Online]. Available: http://ec.europa.eu/information_society/policy/ecomm/radio_ spectrum/_document_storage/com/com-ssa.pdf

[11] A. Vince, "A framework for the greedy algorithm," Discrete Appl. Math., vol. 121, no. 1-3, pp. 247-260, Sep. 2002. [Online]. Available: http://dx.doi.org/10.1016/S0166-218X(01)00362-6

[12] V. V. Shenmaier, "A greedy algorithm for maximizing a linear objective function," Discrete Appl. Math., vol. 135, no. 1-3, pp. 267-279, Jan. 2004. [Online]. Available: http://dx.doi.org/10.1016/S0166-218X(02) 00311-6 